\title{
Percolation study for the capillary ascent of a liquid through a granular soil
}

\author{
Manuel Antonio Cárdenas-Barrantes ${ }^{1, \star}$, José Daniel Muñoz ${ }^{1, \star \star}$, and Nuno Machado Araujo ${ }^{2, \star \star \star}$ \\ ${ }^{1}$ Simulation of Physical Systems Group, Department of Physics, Universidad Nacional de Colombia, Carrera 30 No. 45-03, Ed. \\ 404, Of. 348, Bogota D.C., Colombia. \\ ${ }^{2}$ Centro de Física Teórica e Computacional, Departamento de Física, Faculdade de Ciências, Universidade de Lisboa, Campo \\ Grande, P-1749-016 Lisboa, Portugal.
}

\begin{abstract}
Capillary rise plays a crucial role in the construction of road embankments in flood zones, where hydrophobic compounds are added to the soil to suppress the rising of water and avoid possible damage of the pavement. Water rises through liquid bridges, menisci and trimers, whose width and connectivity depends on the maximal half-length $\lambda$ of the capillary bridges among grains. Low $\lambda$ s generate a disconnect structure, with small clusters everywhere. On the contrary, for high $\lambda$, create a percolating cluster of trimers and enclosed volumes that form a natural path for capillary rise. Hereby, we study the percolation transition of this geometric structure as a function of $\lambda$ on a granular media of monodisperse spheres in a random close packing. We determine both the percolating threshold $\lambda_{c}=(0.049 \pm 0.004) R$ (with $\mathrm{R}$ the radius of the granular spheres), and the critical exponent of the correlation length $v=0.830 \pm 0.051$, suggesting that the percolation transition falls into the universality class of ordinary percolation.
\end{abstract}

\section{Introduction}

Consider a sandpile with its base covered with water. If grains are close enough, water will rise through the interstices of the grains. This capillary rise plays a major role in the transport of fluids across porous media, including water and oil wells. Specially in unsaturated soil structures, like embankments, the capillary rise of water is a real concern, because water can damage the integrity of the structure [1]. The broad spectrum of possible solutions include the use of hydrophobic materials [1] or even the addition of active mechanisms to compensate for the deformations produced by capillary forces [2].

The rising of water through a granular medium is strongly determined by the geometry of the interconnected structure of pores among the grains. First models [3] represented that structure by sites (pore bodies) of arbitrary shape and position interconnected by bonds (pore throats), whose sizes and shapes could be obtained from experimental probes $[4,5]$; many properties, like relative permeability [6] or drainage and imbibition [7] can be estimated from this simplified model. More recent works focus on representing the liquid structures among grains (bridges, menisci and pore bodies) as real as possible, based on experiments (as in X-Ray microtomography [8]) or in computer simulations $[9,10]$. This modeling is able to reproduce water saturation and drying with the water volume as control parameter and to compute forces and pressures [10]. Together with experiments, they have succeeded

\footnotetext{
$\star$ e-mail: macardenasb@unal.edu.co

$\star \star$ e-mail: jdmunozc@unal.edu.co

$\star \star \star$ e-mail: nmaraujo@fc.ul.pt
}

identifying trimers (that is, the junction of three liquid bridges and a meniscus) as the minimal building block to build a pathway for rising water $[8,10]$. The structure itself must strongly change with the critical length of a capillary bridge (a function of contact angle and surface tension [11]), and some studies in two dimensions have been performed to find when a connected structure first appears as either the contact angle [12] or the liquid volume $[13,14]$ increases.

The present work investigates how the set of trimers and enclosing pore bodies at disposal for capillary rising changes from a fully disconnected structure to a connected pathway as the critical length of the capillary bridges increases. The goal is to find both the critical half-length $\lambda_{c}$ for the transition and the critical exponent $v$ for the correlation length. The study is performed on the interstices on three-dimensional random close packings of monodisperse spheres. The capillary model of trimers and pore bodies on random close packings is introduced in Sec. 2 . Next, Sec. 3 analyses the resulting capillary structures by using tools of percolation theory [15-17]. Finally, Sec. 4 summarizes the main conclusions and discussions.

\section{Capillary model}

From a microscopic point of view, water can rise if the grains are close enough to build capillary bridges among them. The shape of a capillary bridge between two identical spherical grains is not strongly affected by gravity, but determined by the contact angle $\theta_{c}$, the liquid-gas surface tension $\gamma$, the liquid volume $V$ and the distance $S$ between 
a)

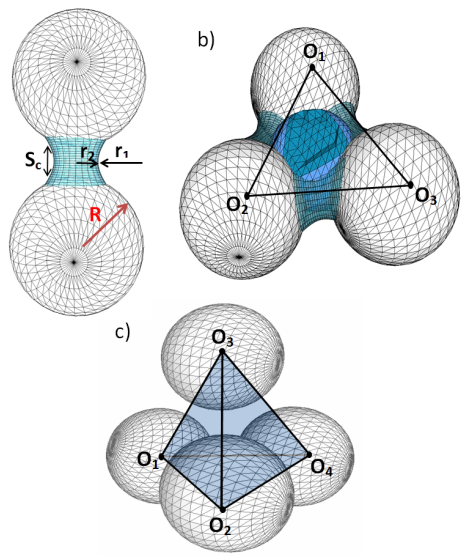

Figure 1. Main capillary structures. (a) Liquid bridge with separation distance $S$. (b) Trimer, built by three liquid bridges (light blue) and a meniscus (dark blue). (c) A liquid volume enclosed by four trimers.

the grains [18]. There is a maximal distance among grains $S_{c}=2 \lambda$ a capillary bridge can overcome. If $S<2 \lambda$, a capillary bridge may eventually established for some $V$; otherwise, there is no possible path between the grains for the water to rise. This limit can be estimated through the toroidal approximation method [19] (Fig. 2) or by numerical simulations.
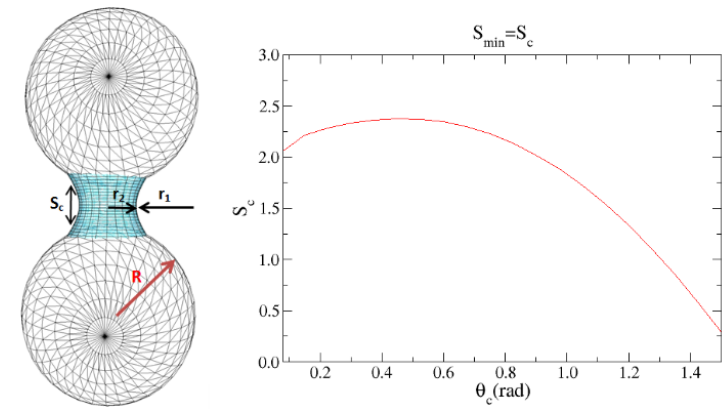

Figure 2. (a) Liquid bridge with separation distance $S$. (b) Maximal separation distance $S_{c}$ for a capillary bridge as function of the contact angle $\theta$ in the toroidal approximation (deduced from data in [11]).

The bridges among grains should be connected in order to build a path for the rising water. From a theoretical point of view, it would be possible to join two bridges with a meniscus; but micro-tomographies on experimental random granular arrays of monodisperse spheres [8] do not show that kind of structure. On the contrary, the minimal connected structures are trimers, that are the junction of three liquid bridges and a meniscus [10] (Fig. 1). A trimer will eventually form for some water content if three grains are so close together that the distances between any two of them are shorter than $S_{c}$ and the angles between every two bridges are smaller than $\pi-2 \theta_{c}$ [11]. Two trimers are assumed connected if they share a bridge, and connected trimers can eventually enclose filling volumes (Fig. 1). Trimers and enclosed volumes form the structure for cap- illary rising. If $S_{c}$ is small, the structure is a disconnected set of clusters. On the contrary, if $S_{c}$ is large enough, there is a connected path across the sample, i.e. a percolating passage for the liquid to rise. The aim of the present work is to characterize the transition across these two regimes driven by $\lambda$, the half maximal length of a capillary bridge - on a monodisperse set of spherical grains by using the standard tools of classical percolation.
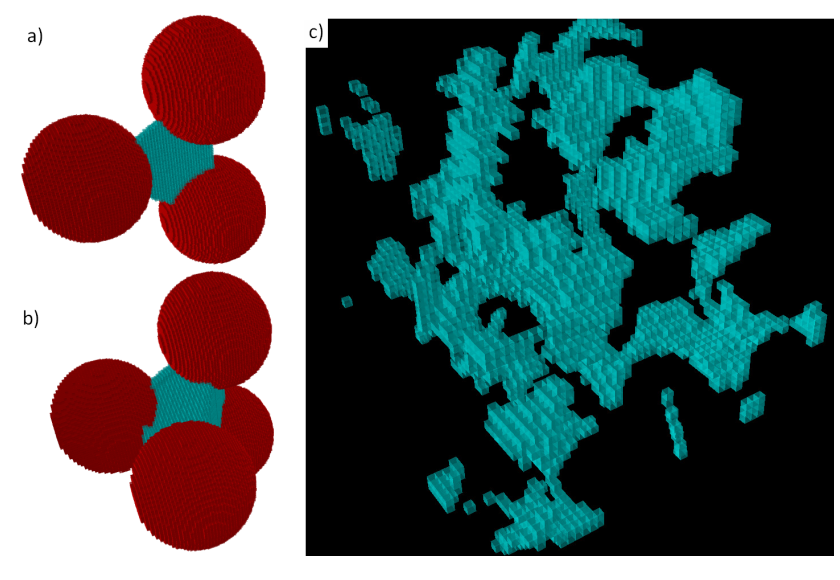

Figure 3. a) Triangle-like liquid structure (trimer). b) Tetrahedron-like liquid structure (enclosed volume). c) Potential capillary rising path (interconnection trimers and enclosed volumes) in a mono-disperse sample of 87 grains with volume fraction $\phi \simeq 0.63$ at a capillary length $\lambda=0.025 R, R$ the radius of the grains (The curve contour do not come from the exact shape of trimers and menisci, but from the removed grains).

\section{Procedure and Results}

The granular medium was modeled as a dense random packing of monodisperse spheres of unitary radius with volume fraction $\phi \approx 0.63$, slightly below the random close packing [20]. The spatial configurations of spheres were generated by using the algorithm of Jodrey and Tory [21] on cubic volumes of sizes $L=22,26,30,36,42,48,56$ and 64, and configurations were accepted if the overlapping lengths between any two spheres was below 0.015 , in units of particle radius. With this procedure, 200 configurations per size for sizes $L \leq 42$ and 500 configurations per size for sizes $L \geq 48$ were generated, corresponding to around 1560 grains each for $L=22$ and 38200 grains each for $L=64$.

For each configuration, we identify sets of three and four grains whose interparticle distances are below $S_{c}=$ $2 \lambda$, and we built triangle-like (trimer) and tetrahedron-like (enclosed volume) structures with those sets of three or four grains, respectively (Fig. 3). The space occupied by the grains themselves is removed. The interconnection of those structures corresponds to possible capillary rising paths. Single bridges are not included, because they are not observed in experiments to contribute to the percolating structure, as discussed in the previous section. The actual shape of the capillary bridges and menisci are not included, because only the connectivity among elements 
is relevant for the percolation exponents, and not the detailed shape of the percolating elements themselves [15]. Then, the whole structure is discretized with a fine grid of cubic cells of side $a=0.031$. Once a value for $\lambda$ was set, each cell was checked to be part of a trimer or an enclosed volume, and trimers become a couple of cells thick. By doing so, the volume that can be occupied by the liquid phase appears as a discrete set of small cubes, as shown in Fig. 3. This allow us to run an efficient site percolation algorithm to study the percolation transition. Two cells are said to be part of the same cluster if they are first neighbors. The Directional Propagation Labelling algorithm (DPL) for GPUs [22] was employed to determine if there was a percolating cluster connecting the lower and upper borders of the cube. It was necessary to implement this algorithm for GPUs because the systems were very large $((800 \times 800 \times 800)$ cells for $L=64)$ and the procedure becomes very time demanding. For instance, the more traditional algorithm by Hoshen and Kopelman [23] takes around 6 hours on a CPU (Intel(R) Xeon(R) CPU E5$2695 \mathrm{v} 3-2.30 \mathrm{GHz}$ ) to evaluate percolation and classify the cluster sizes for a single configuration of system size $\mathrm{L}=36$. By contrast, the DPL algorithm takes only around 15 min to analyze the same configuration on an $\operatorname{Nvidia(R)}$ Tesla(R) K40, what is 25 times faster. The savings in computational time are even larger for larger system sizes. Finally, a bisection algorithm [24] was implemented to determine the effective critical value $\lambda_{c}^{\text {eff }}$ where a percolating cluster first appears on that configuration. The cumulative distribution of these effective critical values for each size are the sigmoids (Fig. 4) that will be the incoming data for the finite-size scaling procedure that follows.

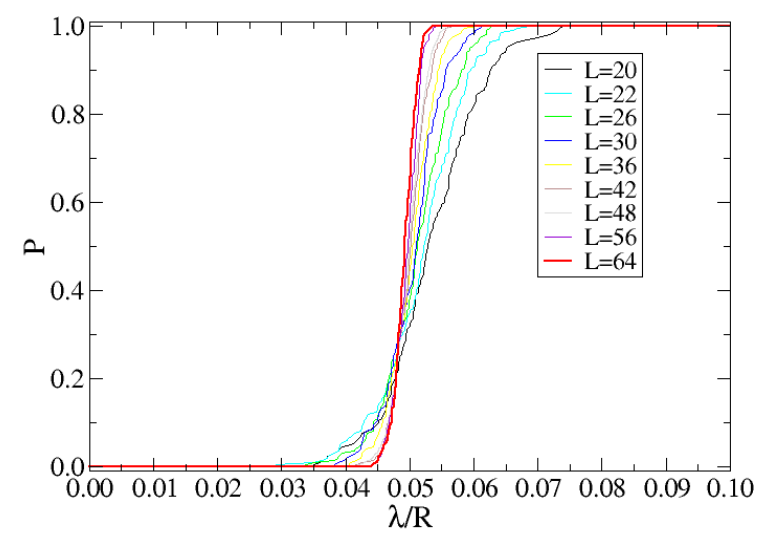

Figure 4. Probability of the capillary structure to percolate as a function of the maximal half-length of a capillary bridge $\lambda$.

The critical exponents and the critical half-length $\lambda_{c}$ for the transition are obtained by the finite-size scaling procedure proposed by Rintoul and Torquato [25]. The scaling procedure starts by fitting the function

$$
f\left(\lambda_{c}^{e f f}, \Delta(L)\right)=\left[1+\tanh \left[\left(\lambda-\lambda_{c}^{e f f}(L)\right) / \Delta(L)\right]\right] / 2
$$

to the cumulative distribution of each size. The effective critical half-length $\lambda_{c}^{\text {eff }}(L)$ and the width $\left.\Delta(L)\right)$ of the distribution obtained from those fittings are plotted against the system size $L$ to obtain the critical parameters of the transition. Assuming that

$$
\Delta(L) \propto L^{\frac{1}{v}}
$$

and plotting $\Delta(L)$ against $L$ (Fig 5) gives the critical exponent $v$ driving the divergence of the correlation length, $\xi \propto\left[\lambda-\lambda_{c}\right]^{-v}$. We obtain $v=0.830 \pm 0.051$. Similarly, plotting $\lambda_{c}^{\text {eff }}(L)$ against $L^{-1 / v}$ (Fig 6) estimates the critical value $\lambda_{c}$ for a system of infinite size, because

$$
\lambda_{c}^{\mathrm{eff}}(L)-\lambda_{c} \propto L^{-\frac{1}{v}}
$$

Our result is $\lambda_{c}=(0.049 \pm 0.004) R$.

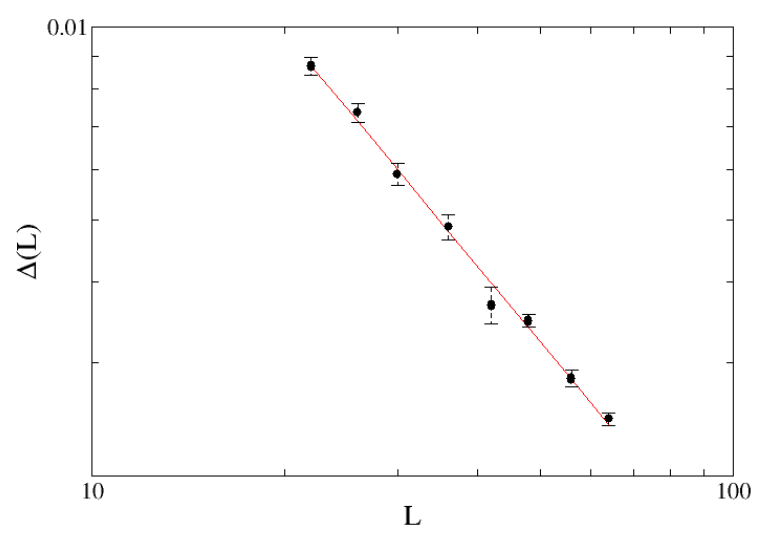

Figure 5. Width of the probability to percolate $\Delta(L)$ as a function of system size $L$. The line is the best power-law fit, with slope $\frac{1}{v}=-1.205 \pm 0.038$.

\section{Conclusions and Discussions}

This work investigates the capillary ascent of a liquid through a granular soil as a percolation transition driven by the half maximal distance between two grains that can be overpassed by a capillary bridge, $\lambda$. Below a critical value $\lambda_{c}$ the structure of trimers and enclosed volumes is disconnected, and no water can rise. Above, the water percolates trough the sample. By using standard techniques of percolation analysis, we found $\lambda_{c}=(0.049 \pm 0.004) R$ and a critical exponent for the correlation length $v=0.830 \pm 0.051$. These results deserve some discussion. First, the finitesize scaling is of very good quality (Fig. 5), suggesting that the technique fits the problem and that there is, indeed, a phase transition. Second, the critical length of a bridge, $2 \lambda_{c}=0.098(6) R$, is less than one half of the mean distance between neighboring grains in the samples (around $(0.258 \pm 0.007) R)$ for a volume fraction $\phi \simeq 0.63)$, which can be considered as a naive a priori estimation of such parameter. Third, the critical exponent $v$ is, within error bars, 


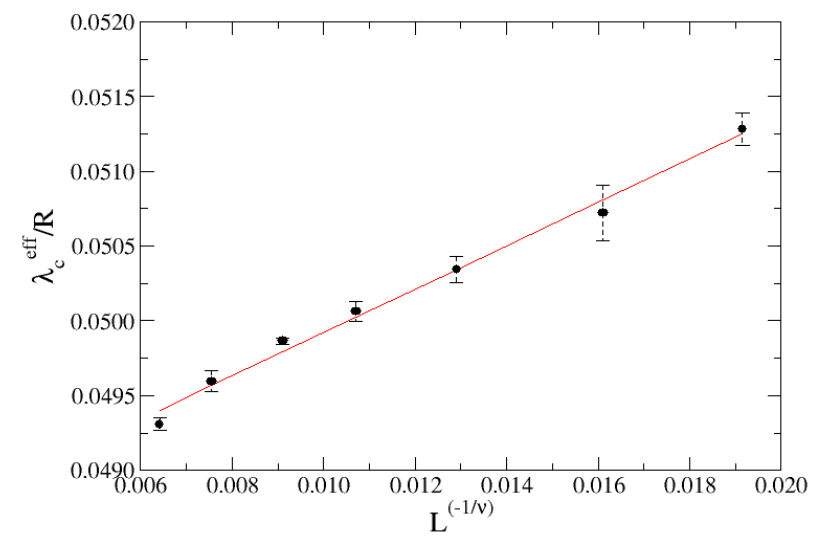

Figure 6. Effective critical half-length of a capillary bridge length $\lambda_{c}^{e f f}(L)$ against $L^{-\frac{1}{v}}$. The linear fit (continuous line) estimates a cut with the vertical axis at $\lambda_{c}=(0.049 \pm 0.004) R$.

the one of ordinary percolation $(v=0.87619(12)$ [26]). This result suggests that each facet among three neighboring grains could turn into a trimer at disposal for the capillary rising almost independently from the other facets, a consequence of the random positions for the grains. This similarity should be confirmed by computing other critical exponents, like the critical exponent $\beta$ for the order parameter, which would be the probability of a cell to belong to the percolating cluster, as usual. Similarly, our study could be reproduced for other void fractions to determine how general are our conclusions. All these are thema for future works.

An usual way to control capillary rising, as we mentioned before, consists in treating some part of the grains with hydrophobic solutions that alter the contact angle, reducing $\lambda$ (Fig. 2). The key question here is which proportion of the material has to be treated to avoid capillary rising. The simulation would proceed in a similar way as the present one, but with three maximal lenghts $\lambda$ : one between untreated grains, other between treated grains and a third one between one treated and one untreated grain. Such a future work will be of great interest in geotechnique.

The present study combines capillary structures and percolation theory to investigate capillary rising through a granular medium. It constitutes a novel approach and a new step in the understanding of this rich phenomenon.

\section{Acknowledgments}

We thanks COLCIENCIAS Young Researchers Program, Grant 2014-645, the Universidad Nacional de Colombia and the Portuguese Foundation for Sciences and Technology (FCT) under Contracts nos. UID/FIS/00618/2013, EXCL/FIS-NAN/0083/2012, and IF/00255/2013 for finnancial support.

\section{References}

[1] N. Khalili, A. Russell, E. Khoshghalb, A, Unsaturated soils : research and applications, $1 \mathrm{st}$ edn. (Crc Press, 2014)

[2] A. Pozzato, A. Tarantino, Unsaturated Soils: Research and Applications 2, 1082 (2014)

[3] I. Fatt, Trans AIME 207, 144 (1956)

[4] H. Vogel, K. Roth, Advances in Water Resources 24, 233 (2001)

[5] M. Coles, P. Hazlett, W. Soll, E. Muegge, K. Jones, Journal of Petroleum Science and Engineering 19, 55 (1998)

[6] U. Fischer, M. Celia, Water Resour. Res. 6, 1089 (1999)

[7] G. Mason, D. Mellor, Journal of Colloid and Interface Science 176, 214 (1995)

[8] M. Scheel, R. Seemann, M. Brinkmann, M. Di Michiel, A. Sheppard, B. Breidenbach, S. Herminghaus, Nature Materials 7, 189 (2008)

[9] S. Motealleh, M. Ashouripashaki, D. DiCarlo, S. Bryant, Transport in Porous Media 99, 581 (2013)

[10] K. Melnikov, R. Mani, F. Wittel, M. Thielmann, H. Herrmann, Cond-mat. Soft 92 (2015)

[11] D. Megias-Alguacil, L. Gauckler, AlChE 55 (2009)

[12] N. Martys, M. Robbins, Phys. Rev. Lett. 66, 1058 (1991)

[13] M. Cieplak, O. Robbins, Phys. Rev. Lett. 60, 2042 (1988)

[14] B. Berkowitz, I. Balberg, Water Resources Research 29, 775 (1993)

[15] D. Staufer, A. Abarony, Introduction to percolation theory, 2nd edn. (Taylor and Francis, 1994)

[16] M. Sahimi, Applications of Percolation Theory, 1st edn. (Taylor \& Francis, UK, 1994)

[17] N. Araujo, P. Grassberger, B. Kahng, K. Schrenk, R. Ziff, The European Physical Journal Special Topics 223, 2307 (2014)

[18] G. Lian, Computer simulation of moist agglomerate colisions (Doctoral Thesis, The University of Aston in Birmingham, 1994)

[19] R. Fisher, J. of Agricultural Science 16, 492 (1926)

[20] M. Shahinpoor, Powder Technology 25, 163 (1980)

[21] J. Jodrey, E. Tory, Physical Review A 32 (1985)

[22] K. Hawick, A. Leist, D. Playne, Parallel Computing 36, 655 (2010)

[23] J. Hoshen, R. Kopelman, Phys. Rev. B 14, 3438 (1976)

[24] W. Press, S. Teukoisky, W. Vetterlingand, B. Flannery, Numerical recipes, 3rd edn. (Cambridge, 2007)

[25] M. Rintoul, S. Torquato, J. Phys. A: Math. Gen. 30, 585 (1997)

[26] X. Xu, J. Wang, J. Lv, Y. Deng, Frontiers of Physics 9, 113 (2014) 